\title{
What keeps sandcastles standing?
}

ny child playing on the beach knows Athat the physical properties of wet and dry sand are very different. Wet sand can be used to build sharp-featured sandcastles that would be unstable in dry sand. We have now quantified the effect of adding small quantities of liquid to a granular medium. Nanometre-scale layers of liquid on millimetre-scale grains dramatically increase the repose angle (the steepest stable slope that the substance can form) and allow the development of long-range correlations, or clumps.

Moisture-induced changes in granular media are primarily caused by adhesive forces associated with interstitial liquid bridges between grains. Such effects are significant in industries as diverse as pharmaceuticals, construction and agriculture. Liquid-induced forces also affect experimental studies of the physics of granular media $^{1-4}$. Previous studies of moisture in granular media have examined only relatively large quantities of water added to highly irregular, porous or water-soluble materials such as coal $^{5,6}$, sugar ${ }^{7}$, seeds or rock chips ${ }^{8,9}$. The resulting data are difficult to analyse and do not aid understanding of the physics of wetted granular media.

We have studied the effects of the addition of small quantities of corn oil and vacuum-pump oil (which both have low vapour pressures) to spherical polystyrene beads, which are insoluble in these oils. We measured the angle of repose by the draining-crater method ${ }^{10}$, with varying draining apertures and liquid content. Our maximum liquid content, (40 times less than the minimum moisture content of previous studies) corresponds to a liquid coating thickness $\left(t_{\text {liq }}\right)$ of less than $50 \mathrm{~nm}$ on the spheres. We found an enormous increase of the angle of repose $\left(\Theta_{\mathrm{R}}\right)$ with $t_{\text {liq }}$ (Fig. 1a). One surprising feature is that $\Theta_{R}$ seems to increase linearly with $t_{\text {liq }}$ up to the point where clumping occurs. The results are independent of oil type and aperture (except for the largest $t_{\text {liq }}$ ), and $\Theta_{\mathrm{R}}$ at $t_{\text {liq }}=0$ agrees with previous measurements by this method $^{10}$.

In dry sand,

$\Theta_{\mathrm{R}}$ is determined by the shape of the grains and by the friction forces. In wet sand, the attractive

forces due to interstitial liquid bridges increase the stability of the
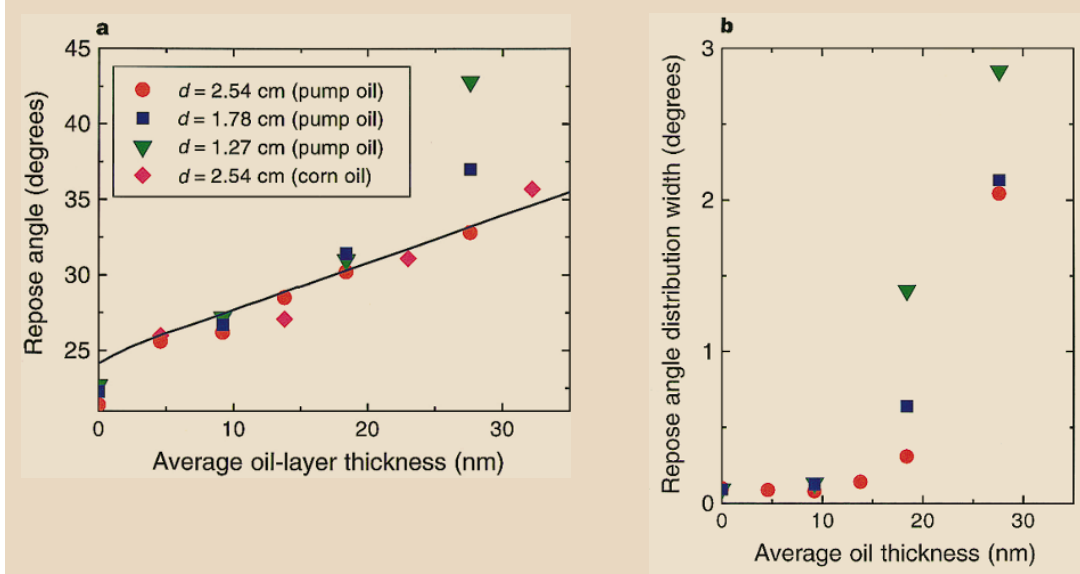

Figure 1a, The angle of repose $\left(\Theta_{R}\right)$ as a function of the average liquid-layer thickness on spheres $(750 \mathrm{~g}$ samples, sphere diameter $0.8 \pm 0.2 \mathrm{~mm}$ ). Draining apertures, $d$, are listed. Data have been corrected for changes in relative humidity (40-50\%) which had a small $\left(\sim 1^{\circ}\right)$ effect on $\Theta_{R}$. Experimental uncertainty is thus roughy $1^{\circ}$. Solid curve, one-parameter theoretical fit. Deviation from the theoretical values for small apertures and large $t_{\text {liq, }}$ is due to clumping. $\mathbf{b}$, The distribution width of measured $\Theta_{R}$ as a function of pump-oil layer thickness. Width corresponds to variations in roughness of the crater surface, which increases with cluster size, as seen with larger $t_{\text {liq. }}$

surface particles and hence $\Theta_{\mathrm{R}}$. Taking into account the observed surface roughness $(\sim 1$ $\mu \mathrm{m})$ of the spheres, we calculated the interparticle adhesive force as a function of liquid content ${ }^{11}$. We then calculated the angle of repose for the wet material using a method based on the stability of the surface particles $^{12}$. This model fits the data with a single free parameter corresponding to the volume of liquid in a typical bridge. The fit indicates that the typical volume of a liquid bridge is $\sim 3 \times 10^{-17} \mathrm{~m}^{3}$ for our maximum $t_{\text {liq }}$, implying that $99.9 \%$ of the liquid does not contribute to the adhesive force, possibly because of the surface roughness.

As liquid was added to the spheres, correlated particle clusters (clumps) formed, the size of which increased with liquid content. The presence of such clusters leads to the appearance of an aperture dependence in $\Theta_{R}$ for the largest values of $t_{\text {liq }}$. The development of clustering is also shown by increases in the width of the distribution of values of $\Theta_{\mathrm{R}}$ with increasing $t_{\text {liq }}$ (Fig. 1b). The width corresponds to variations in the roughness of the crater surface (for $t_{\text {liq }} \gtrsim 20$ $\mathrm{nm}$ the craters were noticeably asymmetrical whereas for smaller $t_{\text {liq }}$ the surfaces were essentially conical with roughness equivalent to a few sphere diameters).

The development of such clusters appears to be rather sudden, suggesting a transition from a situation where the bulk properties are associated with the dynamics of individual grains to a situation where long-range correlations dominate. Our apparatus entirely failed to drain for larger $t_{\text {liq }}(\gtrsim 40 \mathrm{~nm})$ when the size of the clusters approached the aperture size.

Small quantities of wetting liquid can thus dramatically change the properties of granular media, leading to a large increase in the repose angle, clustering and correlation in grain motion. Our results indicate that interstitial liquids can alter many aspects of pattern formation, self-organization $^{1-4}$ and segregation ${ }^{13}$ in granular materials, potentially leading to new physical phenomena not encountered in dry matter.

\section{J. Hornbaker, R. Albert, I. Albert}

\section{A.-L. Barabási, P. Schiffer}

Department of Physics,

University of Notre Dame, Notre Dame,

Indiana 46556, USA

e-mail: peter.schiffer.1@nd.edu

1. Jaeger, H. M., Lui, C.-H. \& Nagel, S. R. Phys. Rev. Lett. 62, $40-43$ (1989).

2. Alanso, J. J. \& Herrmann, H. J. Phys. Rev. Lett. 76, 4911-4914 (1996).

3. Umbanhowar, P. B., Melo, F. \& Swinney, H. L. Nature 382, 793-796 (1996).

4. Jaeger, H. M., Nagel, S. R. \& Behringer, R. P. Rev. Mod. Phys. 68, 1259-1273 (1996).

5. Standish, N., Yu, A. B. \& He, Q. L. Powder Technol. 68, 187-193 (1991).

6. Wolf, E. F. \& von Hohenleiten, H. L. Trans. Am. Soc. Mech. Eng. 67, 585-599 (1945).

7. Craik, D. J. \& Miller, B. F. J. Pharm. Pharmaceut. 10, 136T-144T (1958).

8. Fowler, R. T. \& Wyatt, F. A. Aus. J. Chem. Eng. 1, 5-8 (1960).

9. Pilpel, N. Manufact. Chem. Aerosol News 41, 19-22 (1970).

10. Brown, R. L. \& Richards, J. C. Principles of Powder Mechanics (Pergamon, Oxford, 1970).

11.Eremenko, V. N., Nadich, Yu. V. \& Lavrimenko, I. A. LiquidPhase Sintering (Consultants Bureau, New York, 1970).

12. Albert, R., Albert, I., Hornbaker, D. J., Schiffer, P \& Barabási, A.-L. Phys. Rev. Lett. (submitted).

13. Makse, H. A., Havlin, S., King, P. R. \& Stanley, H. E. Nature 386, 379-382 (1997). 